\title{
In Search of Novel Targets for Heart Disease: Myocardin and Myocardin-Related Transcriptional Cofactors
}

\author{
Alexander T. Mikhailov and Mario Torrado \\ Institute of Health Sciences, University of La Coruña, Campus de Oza, Building El Fortin, Las Jubias Street s/n, \\ La Coruña 15006, Spain \\ Correspondence should be addressed to Alexander T. Mikhailov, margot@udc.es
}

Received 19 January 2012; Accepted 5 March 2012

Academic Editor: Jeffrey R. Moore

Copyright ( 12012 A. T. Mikhailov and M. Torrado. This is an open access article distributed under the Creative Commons Attribution License, which permits unrestricted use, distribution, and reproduction in any medium, provided the original work is properly cited.

Growing evidence suggests that gene-regulatory networks, which are responsible for directing cardiovascular development, are altered under stress conditions in the adult heart. The cardiac gene regulatory network is controlled by cardioenriched transcription factors and multiple-cell-signaling inputs. Transcriptional coactivators also participate in gene-regulatory circuits as the primary targets of both physiological and pathological signals. Here, we focus on the recently discovered myocardin-(MYOCD) related family of transcriptional cofactors (MRTF-A and MRTF-B) which associate with the serum response transcription factor and activate the expression of a variety of target genes involved in cardiac growth and adaptation to stress via overlapping but distinct mechanisms. We discuss the involvement of MYOCD, MRTF-A, and MRTF-B in the development of cardiac dysfunction and to what extent modulation of the expression of these factors in vivo can correlate with cardiac disease outcomes. A close examination of the findings identifies the MYOCD-related transcriptional cofactors as putative therapeutic targets to improve cardiac function in heart failure conditions through distinct context-dependent mechanisms. Nevertheless, we are in support of further research to better understand the precise role of individual MYOCD-related factors in cardiac function and disease, before any therapeutic intervention is to be entertained in preclinical trials.

\section{Introduction}

Heart failure (HF) is the common end-stage condition of various cardiovascular disorders characterized by a progressive decrease in cardiac output combined with insufficient or absent compensatory mechanisms. HF is a major public health problem affecting 15 million patients in the European Union [1]. In descriptive terms, HF can be classified as acute or chronic, congestive, systolic or diastolic, with high or low output, left sided or right sided, and backward or forward. Prevalent causes of sporadic (nonfamilial) HF include myocardial infarction, hypertension, and ischemic or dilated cardiomyopathy. Diuretics, ACE (angiotensinconverting enzyme) inhibitors, and angiotensin-II receptor blockers improve survival in patients with chronic HF but do not prevent cardiomyocyte molecular and structural deterioration (reviewed in [2]). Despite progress in both clinical investigation and basic cardiovascular research (see
[3-5]), targeting the molecular mechanisms that promote and sustain HF development in patients still remains elusive (albeit with certain progress, see $[6,7]$ ).

Extensive research over the past 10 years has begun to provide significant advances in our understanding of the interplay between heart development and disease [8-11]. The important step in this direction is illustrated by recent studies of gene regulatory networks (GRNs) operating in cardiac muscle cells [12-15]. The unraveling of GRNs in both normal and diseased heart offers the opportunity to identify single key factors for regulation of the gene expression networks which are ubiquitously altered in failing myocardium, regardless of the etiology of HF [16].

The major players involved in cardiac GRNs are transcription factors, notable among which is the serum response factor (SRF; [15]). SRF, a member of the MADS (MCM1, $A G, D E F A, S R F$ ) box family of transcription factors, binds 
as a dimer to specific sites (known as CArG boxes located within serum response elements) on DNA driving the expression of hundreds of target genes in a wide array of cell types and tissues, from brain to cardiac muscle [17-19]. Although ubiquitously expressed, SRF plays specialized roles in different cellular environments. One of the mechanisms by which SRF regulates gene expression in a cell-specific manner is through SRF recruitment of several cofactors which are specifically expressed in a given cell type. In different muscle cell types (cardiac, smooth, skeletal), cell-specific regulation of the SRF-dependent gene expression is achieved by SRF binding of specific coactivators, such as myocardin (MYOCD) and myocardin-related transcription (MRTF) cofactors, MRTFA and MRTFB (reviewed in [20-23]).

The data from both animal models and clinical research in patients provided evidence of pathological consequences of both SRF redundancy and deficiency in the adult heart. In fact, SRF hyperexpression can lead to pathological hypertrophy, while inhibition of SRF activity can result in development of dilated cardiomyopathy (reviewed in [18]). In light of the involvement of SRF in adverse cardiac remodeling, it has been of interest to explore the potential contributions of MYOCD family members, as SRF coactivators, to heart disease. As a result, the data accumulated over the last years clearly implicate the MYOCD protein family in several common forms of adult cardiac disease, such as pathological cardiac hypertrophy, myocardial infarction, and HF [24]. Moreover, the MYOCD gene expression is upregulated in circulating blood cells of patients with sporadic hypertrophic cardiomyopathy [25] or hypertension [26].

There have been a series of excellent reviews on molecular and functional characterization, and regulation of MYOCD and MRTFs in different muscle and nonmuscle cell types [20, 24, 27-29], and these essential facets will be commented relatively briefly here. Instead, this paper emphasizes recent insights into the involvement of transcriptional cofactors of the MYOCD family in cardiac dysfunction and to what extent experimental modulation of these factors' expression in vivo can correlate with cardiac disease outcome.

\section{Myocardin Family Proteins: Functional Domains and Transcription Factor Binding Motifs}

The myocardin protein family includes (as mentioned above) MYOCD itself and two MYOCD-related transcription factors, MRTF A and B (also referred to as MAL/MKL1 and MAL16/MKL2, resp.). Properties of the MYOCD-related family of SRF-binding cofactors have been characterized most extensively in MYOCD itself, as the founding member, and are presumed to be shared among other MYOCD family members. Although sometimes members of the MYOCD protein family are called "transcription factors" (see, e.g., [24]), they do not bind to DNA itself. Instead, they form a ternary complex with SRF anchored to the CArG box of promoters of cardiac and smooth muscle (SM) contractile genes.
MYOCD is a SAP (scaffold-attachment factor A/B, Acinus, PIAS) domain protein that was discovered by Olson's group during an in silico search for genes underlying early heart development [30]. Mrtf- $A$ and $M r t f-B$ were initially isolated by Wang and colleagues [31] on screening cDNA libraries with Myocd-related probes. All of them show a similar domain organization (Figure 1) consisting of RPEL motifs, a basic domain, a glutamine-rich region, a SAP domain, a leucine zipper-like region, and a transcription activation domain.

The N-terminal region of MYOCD proteins contains several RPEL motifs (known also as RPEL domain) capable of interacting with globular actin. Differences between RPEL domains appear to define different nucleocytoplasmic transport activities of MYOCD family proteins: MYOCD is constitutively located in the nucleus whereas its family members, MRTF-A and MRTF-B, mostly reside in the cytoplasm and translocate to the nucleus in response to actin polymerization (for more details see $[37,38]$ ). Recently, it has been shown that nuclear accumulation of MYOCD family members also depends on their different relative affinities for nuclear import factors $[39,40]$. Of note, the MEF2 (myocyte enhancer factor 2) binding motif located in RPEL1 is unique to MYOCD; transfer of this MEF2 binding motif to MRTFA confers the ability to co-activate the MEF2 transcription factor [32]. The other SAP domain protein (named MASTR) shares this motif with MYOCD and acts as a MEF2 coactivator [41].

MRTF-A and MRTF-B share strong sequence homology with MYOCD in the basic and Q-rich domains, which are responsible for SRF binding. The SAP domain, characteristically observed in diverse nuclear proteins involved in chromatin organization/remodeling, is not required for interaction of MYOCD proteins with SRF. However, mutations in the SAP domain of MYOCD affect activation of some SRF-dependent gene targets, but not others, suggesting a role of this domain in target gene discrimination. Although all MYOCD proteins have a highly conserved transactivation domain at their C-terminal region, MRTF-A activates CArGdependent SM-gene promoters reaching levels similar to those induced by MYOCD itself, whereas MRTF-B is less effective in this regard. Several consensus regions of MYOCD have been shown to be putative sites for binding of transcription factors, other than SRF, which are also involved in regulation of cardiac gene transcription and expression or associated with the response of cardiac tissue to stress (see Figure 1, MEF-2, TBX5, GATA4, and NKX3.1). Forthcoming studies should reveal the full potential of the MYOCD family in cardiac transcription regulatory networks.

Formally viewed, a high degree of domain similarity between members of the MYOCD family indicated they could have similar or overlapping functions in developing and adult heart. However, evidence of similar domain organization alone does not prove that MYOCD family members do in fact perform identical or similar functions or actions in the heart. Basic assumptions regarding similarities and differences in their expression and functions in cardiac tissue are discussed next. 


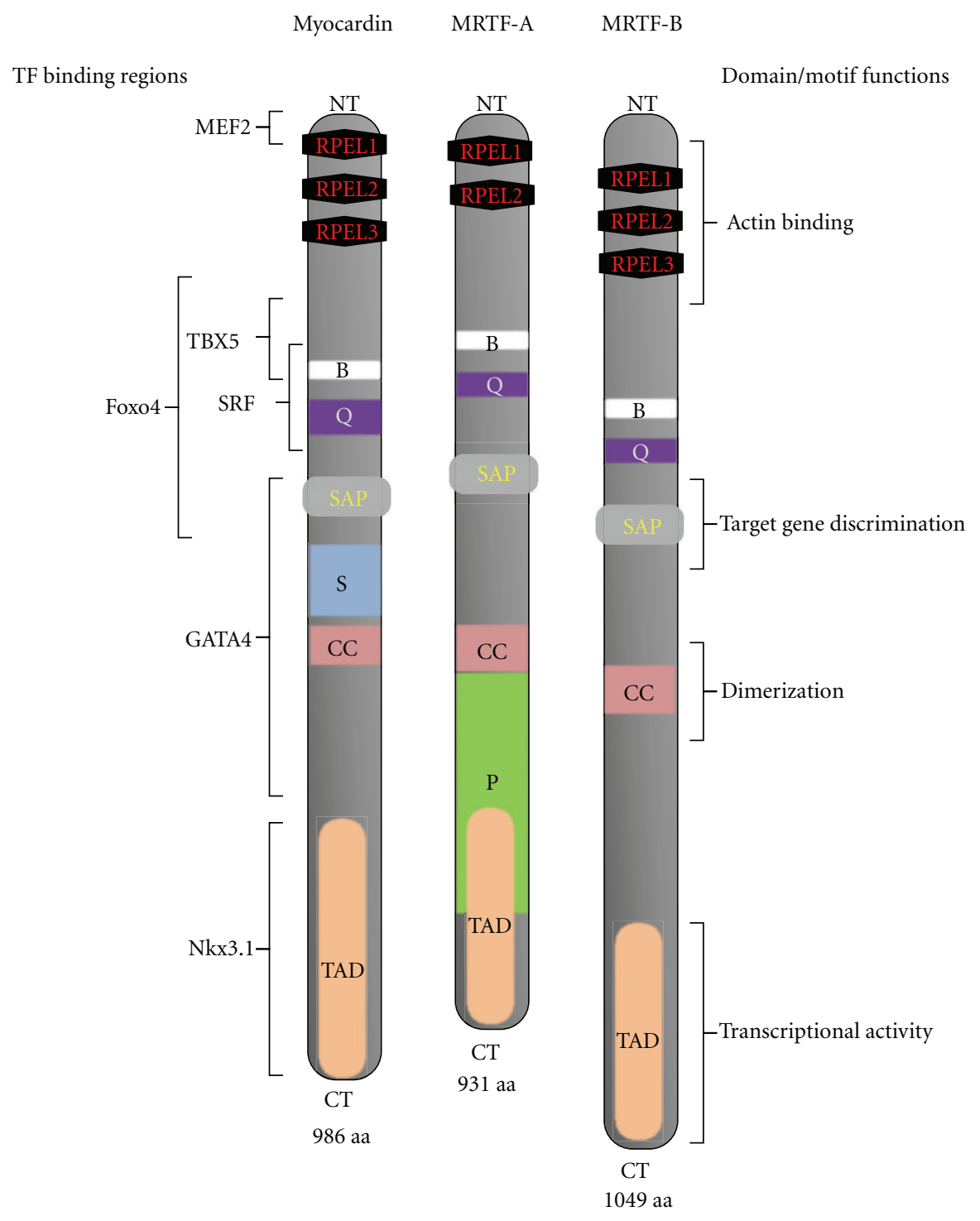

FIGURE 1: The myocardin family of transcriptional cofactors: protein signatures, domains, and functional sites. The domain/motif structures of the human proteins are shown: RPEL 1-3 (RPEL domain), B: basic domain, Q: glutamine-rich region, SAP: SAP domain; S: serine-rich region; CC: coiled-coil (leucine zipper-like) motif; P: proline-rich region; TAD: transcription activation domain. The regions essential for binding of transcription factors (TF). MEF2: myocyte enhancer factor-2 [32], TBX5: T-box transcription factor 5 [33], FOXO4: forkhead box protein O4 [34], GATA-4: member of GATA family of zinc finger transcription factors [35], NKX-3.1: homeobox NK-3 transcription factor-1 [36]. NT/CT: amino/carboxyl terminus.

\section{Myocardin Family Proteins and Heart Development: Lessons from Mouse Knockout Models}

Complete loss-of-function (total/global or constitutive gene knockout) experiments suggest the function(s) of a gene of interest through the phenotype that results from its deficiency in mouse mutants and, by extrapolation, in humans. Even an early embryonic lethal phenotype might be informative enough to predict a gene as essential for a given fetal tissue/organ, but roles the gene may play later in development as well as in postnatal/adult life remain unknown. To overcome these problems, techniques have been developed to create conditional knockout models, in which a gene of interest can be inactivated in a spatially and temporally controlled fashion. Several such approaches have been used to evaluate to which extent the members of the MYOCD family of transcriptional cofactors are involved in molecular and cellular processes underlying heart development and maturation.

The members of the MYOCD family are coexpressed in cardiac as well as different SM lineages during early mouse embryonic development $[30,31]$. Although a single gene-null mutation in Myocd and $M r t f-B$ resulted in a wide range of tissue abnormalities and embryonic lethality, here we will consider only structural and functional 
consequences of inactivation of these genes in the heart and associated structures. (For accounts of the wider spectrum of perturbations observed in these knockouts, see references provided in Table 1).

In mice, the complete loss of Myocd leads to a severe early developmental, lethal phenotype from a failure in vascular SM differentiation [42]. However, early cardiogenesis was unaffected in the Myocd-null mice. Heart development in Myocd knockouts could be partially rescued by redundant activities of $M r t f-A$ and/or $M r t f-B$. Although repeatedly stated, this suggestion has not yet been tested experimentally. By contrast, despite the fact that both Myocd and Mrtf-B are coexpressed in embryonic vascular SM cells, Myocd-null mice lack differentiated SM cells in the dorsal aorta and placental vasculature.

Due to the early lethal phenotype of the Myocd-null embryos, a conditional knockout approach was taken for selective ablation of Myocd in developing heart. Cardiorestricted inactivation of the Myocd gene did not alter heart development. However, after birth mutant mice with a conditionally inactivated Myocd gene develop dilated cardiomyopathy (DCM) accompanied by impaired cardiomyocyte structural organization and severely depressed systolic function [43]. In chimeric Myocd knockout mice, generated by injection of Myocd-null embryonic stem cells into wide-type blastocysts, Myocd-null cells fail to contribute to formation of ventricular myocardium, although these cells were phenotypically normal and did not display ultrastructural alterations [50]. Moreover, these results imply that Myocd is absolutely required for functional development of ventricular myocardium and can play a pivotal role in the response of the heart to stressful stimuli after birth.

In contrast to Myocd-null embryos, Mrtf-B homozygous null or hypomorphic knockout embryos express cardiovascular phenotypic abnormalities resembling those observed in human patients with congenital heart disease. Although knockout models used in these studies resulted in phenotype of differing severity, thin-walled ventricular myocardium and ventricular septal defects were observed in each model setting. Abnormal patterning of the branchial arch arteries [47] and truncus arteriosus [48, 49] are also among the other common manifestations (see Table 1). Although the consequences of depleting $M r t f-B$ specifically within the myocardium have not yet been reported, the results of global Mrtf-B knockouts demonstrate that this factor is absolutely required for embryonic heart development, specifically within ventricular and cardiac outflow tract compartments.

The role of $M r t f-A$ in cardiovascular development is still unclear. Two groups have independently shown that Mrtf$A$ knockouts are viable and can reach adulthood without obvious cardiac or other organ abnormalities; only knockout females possess mammary gland dysfunction due to the failure of mammary myoepithelial cells to differentiate [44, 46]. Although $M r t f-A$ is not absolutely required for heart development, it may play a particular role in the survival of myocardial cells during certain periods of development: a set of $M r t f-A$ null mouse embryos suffered dilatation of all cardiac chambers and died at midgestation stages [46]. In addition, young adult mice lacking $M r t f-A$ show a significantly weaker hypertrophic response to both acute and chronic pressure overload suggesting $M r t f-A$ is involved in hypertrophy signaling pathways in the postnatal heart $[45,51]$.

Mice lacking each member of the Myocd family exhibit distinct global phenotypes during development [24, 28]. Within the cardiovascular system, knockout mutations in the Myocd, Mrtf-A, or Mrtf-B gene specifically affect distinct aspects of heart formation at different stages of development. Inactivation of $M r t f-B$ drastically alters pattern formation in the developing heart that can lead to congenital heart malformations, whereas deficiency in Myocd and Mrtf-A becomes phenotypically evident in rather mature heart resulting, respectively, in development of severe DCM or poor hypertrophic response. Whether these factors are also involved in adult heart function and, if so, how these factors modulate the responsiveness of the heart to stress signaling is highlighted below.

\section{Myocardin Family Proteins in Adult Heart}

Once heart development is completed, postnatal functional maturation of the heart takes over extending the use of cardiac GRNs to adulthood raising the possibility that some forms of adult heart disease can result from adult-related alterations in components of these regulatory pathways [52$55]$.

Members of MYOCD gene family are expressed in normal and diseased adult myocardium. The ventricular myocardium, a highly ordered tissue structure, consists of different cell types (cardiac myocytes, fibroblasts, and vascular endothelial and SM cells) which all are involved in myocardial remodeling in response to physiological and pathological stresses. Here, we will discuss arguments which suggest the possible functions of MYOCD family factors in both cardiomyocyte and fibroblast cell compartments of adult myocardium (Figure 2).

4.1. MYOCD. MYOCD is absolutely required for maintenance of adult heart function. Cardio-restricted inactivation of the Myocd gene in the adult mouse heart resulted in development of severe four-chambered DCM as a result of massive myocyte loss via apoptosis and replacement fibrosis. $M r t f-B$, showing nonaltered cardiac expression, did not rescue the DCM phenotype in Myocd-knockdown mice. It was found in this model system that Myocd itself functions in a cardiomyocyte autonomous manner to regulate myocyte gene expression and myofibrillar organization and to prevent cardiomyocyte apoptosis in response to physiological hemodynamic stress [43].

In light of these results, it is not surprising that MYOCD has previously been implicated in remodeling of ventricular myocardium in response to either physiological or pathological pressure-overload hypertrophy [5658]. In addition, MYOCD is necessary for and mediates the hypertrophic response of ventricular myocardium to a variety of stimuli such as phenylephrine, endothelin, isoproterenol, aldosterone, angiotensin II, insulin and insulin-like 
TABLE 1: Mouse loss-of-function studies of Myocd-related family.

\begin{tabular}{|c|c|c|c|c|c|}
\hline Gene & knockout & Embryonic cardiovascular phenotype & Embryonic phenotype & After-birth phenotype & Reference \\
\hline Myocd & Constitutive & $\begin{array}{l}\text { Block in SMC differentiation; heart } \\
\text { development is obviously normal }\end{array}$ & $\begin{array}{l}\text { Lethal; } \\
\text { early-to-mid-gestation }\end{array}$ & No survivors & {$[42]$} \\
\hline Myocd & $\begin{array}{l}\text { Conditional, } \\
\text { heart } \\
\text { restricted }\end{array}$ & Obviously normal heart development & Obviously normal & DCM, HF & {$[43]$} \\
\hline \multirow{2}{*}{$M r t f-A$} & \multirow{2}{*}{ Constitutive } & \multirow{2}{*}{ No obvious abnormalities } & \multirow{2}{*}{ Obviously normal } & $\begin{array}{l}\text { Myoepithelial defects } \\
\text { of the mammary gland; }\end{array}$ & {$[44]$} \\
\hline & & & & $\begin{array}{l}\text { poor hypertrophic } \\
\text { response }\end{array}$ & {$[45]$} \\
\hline \multirow[b]{2}{*}{$M r t f-A$} & \multirow{2}{*}{ Constitutive } & 65\%-no obvious abnormalities; & $65 \%$-obviously normal & \multirow{2}{*}{$\begin{array}{l}\text { Myoepithelial defects } \\
\text { of the mammary gland }\end{array}$} & \multirow[b]{2}{*}[46]{} \\
\hline & & $35 \%$-dilated heart & $\begin{array}{l}\text { 35\%-lethal, } \\
\text { early-to-mid-gestation }\end{array}$ & & \\
\hline$M r t f-B$ & Constitutive & $\begin{array}{l}\text { Double outlet RV, ventricular septal defects, } \\
\text { thin-walled myocardium, abnormal } \\
\text { patterning of the branchial arch arteries }\end{array}$ & Lethal; midgestation & No survivors & {$[47]$} \\
\hline$M r t f-B$ & Constitutive & $\begin{array}{l}\text { Double outlet RV, cardiac outflow tract } \\
\text { defects, ventricular septal defects, persistent } \\
\text { truncus arteriosus }\end{array}$ & Lethal, late gestation & No survivors & {$[48]$} \\
\hline$M r t f-B$ & Constitutive & $\begin{array}{l}\text { Ventricular septal defects, thin myocardium, } \\
\text { truncus arteriosus }\end{array}$ & Lethal; late gestation & $\begin{array}{l}\text { Only a few grossly } \\
\text { normal survivors }\end{array}$ & {$[49]$} \\
\hline
\end{tabular}

RV: right ventricle; HF: heart failure; DCM: dilated cardiomyopathy.

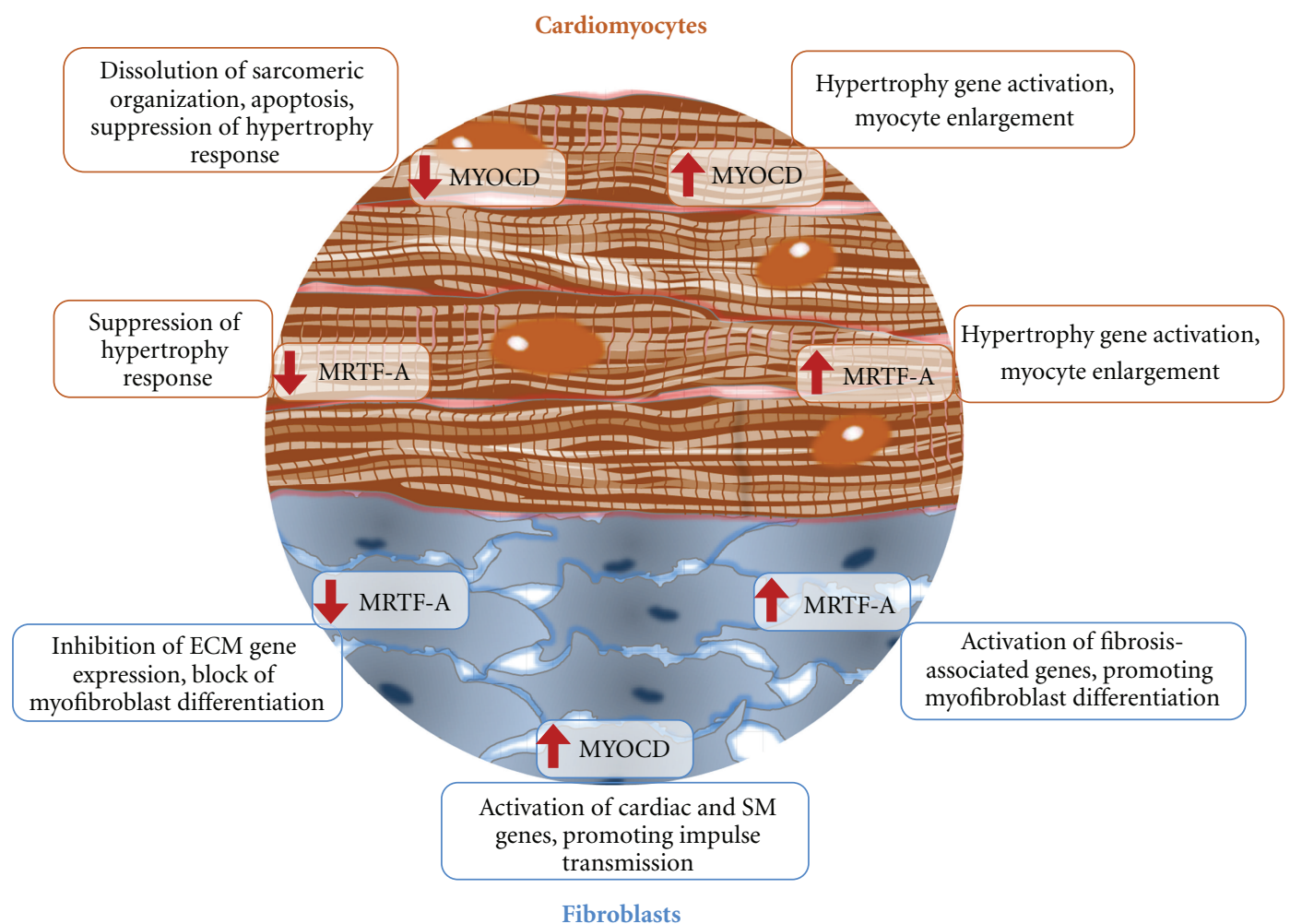

FIgURE 2: Dissecting the cell-autonomous roles of myocardin and MRTF-A in adult ventricular myocardium. Red arrows indicate upor downregulation. In cardiomyocytes, forced expression of either Myocd or Mrtf-A induces hypertrophic gene expression and myocyte enlargement, whereas inhibition of any of these factors markedly attenuates hypertrophic responses. Although both factors display prohypertrophic activities, MYOCD, but not MRTF-A, is absolutely required for myocyte structural integrity and survival [43]. In cardiac fibroblasts, forced expression of $M r t f-A$ activates profibrosis gene expression and myofibroblast differentiation, whereas a loss of functional Mrtf- $A$ leads to opposite effects. Forced Myocd expression stimulates both SM (including markers of myofibroblast differentiation) and cardiac genes (including cardiac ion channels and connexins) in ventricular fibroblasts. 
growth factors, and beta-transforming growth factor (TGF$\beta$ ). Forced expression of Myocd in cultured cardiomyocytes increases cell size and expression of molecular markers of cardiac hypertrophy, whereas knockdown of Myocd attenuates hypertrophic response capacity of cardiomyocytes [58-63]. Hypoxia in neonatal cardiomyocytes increases levels of MYOCD and ROS (reactive oxygen species) resulting in cellular hypertrophy, which can be partly reversed by atorvastatin treatment [64]. Collectively, the experimental data indicate at least two important roles for MYOCD in adult cardiomyocytes: its role in maintenance of structural integrity and survival of cardiomyocytes and its role as a prohypertrophic factor in hypertrophic remodeling induced by genetic models, aortic constriction, and neurohumoral factors.

Despite the popular assumption that MYOCD is expressed almost exclusively in cardiac and SM cells, MYOCD expression was also detected in human fibroblasts [65] where it is involved in functional differentiation and has a negative role in cell proliferation [66]. In fact, in model cell-based assays overexpression of Myocd resulted in inhibition of cell-cycle progression at the $\mathrm{G} 2 / \mathrm{M}$ phase and formation of polyploidy cells [67]. MRTF-A and MRTF-B also exert antiproliferative effects on fibroblasts [68].

Expression of Myocd in mouse cardiac fibroblasts has not been detected [69] but rat cardiac fibroblasts cultured in a medium conditioned by mesenchymal stem cells (MSCs) did express Myocd [70]. In rat cardiac fibroblasts, Myocd expression appears to be age dependent and influenced by hypoxia-inducible factor $1 \alpha$ [71].

It has been demonstrated that MYOCD-mediated transactivation of target genes is promoter and cell context dependent [72, 73]. MYOCD alone was not sufficient to activate cardiac-specific genes in pluripotent 10T1/2 fibroblasts [74, 75]. Ectopic Myocd expression in skeletal muscle-like $\mathrm{BC} 3 \mathrm{H} 1$ cell line induced cardiac and SM genes and stimulated formation of SM-like filaments with no evidence for cardiac sarcomerogenesis [76]. Infection of human epicardial cells with an adenovirus vector encoding Myocd co-activated both SM and cardiac marker genes but did not lead to the assembly of SM-like filaments [77]. Similarly, forced Myocd expression resulted in the activation of a broad range of cardiomyocyte and SM genes in both human foreskin-derived [65] and myocardial scar (MSFs) fibroblasts [78]. Although such MYOCD-forced MSFs did not acquire the cardiomyocyte structural and functional phenotype, they became capable of conducting an electrical impulse. In vitro, placing of Myocd-forced fibroblasts between two separate cardiomyocyte fields resulted in resynchronization of two dyssynchronously beating cardiomyocyte areas [78]. Of note, forced expression of Myocd enhances the therapeutic effect of MSCs implanted into the infracted area of postmyocardial infarction (MI) in mice [79].

Myocd expression is upregulated by TGF- $\beta 1$ in both fibroblasts [66] and SM cells [80], and MYOCD participates in TGF- $\beta 1$ signal-transducing pathways to activate SM gene transcription $[81,82]$. Cardiac TGF- $\beta 1$ expression is upregulated in both animal models of MI and ventricular hypertrophy and patients with hypertrophic or DCM [83]. Although unproven as yet, it is tempting to speculate that in the infarcted heart TGF- $\beta 1$ induction of Myocd expression might contribute to fibroblast-to-myofibroblast transition. In this sense, a very recent report provides direct evidence that both MRTF-A and MRTF-B are key regulators of TGF$\beta 1$-induced fibroblast-to-myofibroblast transition [84].

4.2. MRTF-A. Consistent with its discovery as a MYOCDrelated factor, it has initially been suggested that MRTF-A plays a potentially important role in the heart [31]. However, evidence of this has been found only recently with the use of cardiac dysfunction models in adult Mrtf-A null mice. It is appropriate to keep in mind that under basal conditions Mrtf-A null mice did not manifest any obvious cardiac structural or physiological deficiencies [44], and inactivation of Mrtf-A expression was not associated with changes in Myocd or Mrtf-B expression in mutant hearts [45].

In vivo studies, using the aortic banding model, indicated that loss of Mrtf-A inhibits or diminishes activation of the hypertrophic gene program induced in left ventricular (LV) myocardium by mechanical pressure overload. At the morphological level, a degree of LV hypertrophy was also decreased in Mrtf-A mutant mice with experimentally induced chronic pressure overload as compared to controls. The same effects were also observed in Mrtf-A-null mice subjected to chronic angiotensin II treatment [45]. Taken together, the results identified MRTF-A as a factor mediating prohypertrophic signaling evoked by both mechanical stress and neurohumoral stimulation in ventricular myocardium.

However, it remained uncertain if MRTF-A itself functions in a cardiomyocyte autonomous manner to regulate ventricular hypertrophy. Recent experiments using cultured neonatal cardiomyocytes have demonstrated that overexpression of $M r t f-A$ does induce hypertrophic growth and expression of cardiac hypertrophy marker genes in these cells. In addition, it was found that the expression of both Mrtf-A and Myocd is induced by hypertrophic signals, whereas dominant-negative mutants of these factors (lacking TAD domain, see Figure 1) attenuate agonist-induced cardiomyocyte hypertrophy. Also, inhibition of endogenous Mrtf- $A$ expression reduced phenylephrine, angiotensin-II, and TGF- $\beta$-induced hypertrophy in neonatal cardiomyocytes [63]. These data, together with those of Mrtf-A null mice $[45,51]$, indicate that besides MYOCD, MRTFA can play an important role in cardiac hypertrophy as a myocardial prohypertrophic factor.

In the adult heart, $M r t f-A$ is robustly expressed in normal cardiac fibroblasts which are activated to become SM-like myofibroblasts in response to MI. Myofibroblasts strongly express $\alpha$-SM actin and extracellular matrix (ECM) proteins. In the recent report [69], MIs were generated using Mrtf$A$ null and wide-type adult mice by surgical ligation of the left anterior descending coronary artery. Both, a significantly reduced infarct size and attenuated upregulation of the ECM markers (collagens, elastin) in the border zone, were observed in MI Mrtf-A null compared to MI wide-type mice. The fibroblast-to-myofibroblast transition is believed to be 
primarily responsible for fibrotic remodeling of the postMI heart. The expression of SM markers of myofibroblast activation, as well as a density of myofibroblasts in the MI border zone, was attenuated in Mrtf-A-null mice.

Also, Mrtf- $A$ null mice display reduced myocardial fibrosis in response to angiotensin treatment. Forced expression of $M r t f-A$ in isolated cardiac fibroblasts was sufficient to activate myofibroblast-associated SM genes that were downregulated in MI Mrtf-A null hearts [69]. These results suggest that MRTF-A can play an essential role in promoting fibroblastto-myofibroblast transition and fibrotic remodeling in the post-MI heart. (For more detailed comments and nuances related to MRTF-A-regulated pathways of myofibroblast activation, see [85]).

\section{Myocardin Family Proteins and Heart Disease}

As mentioned above, many lines of evidence have implicated MYOCD as an important regulator of hypertrophic growth of the heart. However, it was not until recently that the ability of MYOCD to promote cardiac hypertrophy in vivo was directly examined. In neonatal piglets, in vivo forced expression of MYOCD in ventricular myocardium resulted in activation of a set of fetal cardiac and SM genes associated with impaired systolic performance although no significant change in heart-to-body weight ratio was detected in MYOCD-transfected compared with control-sham animals [86]. In neonatal piglets, the right (RV) and left (LV) ventricles display such a different degree of hypertrophy that the RV can be used as a slower heart-matched referencecontrol for the much more rapidly thickening $\operatorname{LV}[87,88]$. Nevertheless, expression of $M Y O C D$ was found to be similar in both the LV and RV of porcine as well as human neonates [56]. The simplest interpretation of these data is that although MYOCD does upregulate the expression of a set of SRF/MYOCD-dependent genes in response to hypertrophy signals, it alone is not sufficient to discriminate among different patterns of LV/RV remodeling in the adult heart in vivo.

In addition to its role in adaptive gene expression and maintenance of cardiac function, MYOCD has also been implicated in the response of the adult heart to pathological stresses during severe hypertrophic remodeling $[58,89]$ and at end-stage HF due to dilated, ischemic, and cardiotoxic $\mathrm{CM}[56,58,90]$. All of these conditions are characterized by upregulation of MYOCD expression in failing LV myocardium. In addition, MYOCD SM targets are also upregulated in failing myocardium in both animal models and patients [91]. Although this correlative evidence links MYOCD signaling to the acquisition of pathological conditions, the role that MYOCD gene activation plays in $\mathrm{HF}$ conditions in vivo has remained unclear. Recently, we attempted to integrate upregulated MYOCD signaling into the pathogenesis of HF, using targeted RNAi-mediated $M Y O C D$ gene inhibition in the porcine model of diastolic HF (DHF). In vivo silencing of endogenous MYOCD at mid-advanced stages of DHF resulted in downregulation of MYOCD-dependent SM-gene expression in failing myocardium [92]. Such adjusting of MYOCD and SM-target expression levels to the range of physiological variation resulted in restoring diastolic function and extending the survival of failing animals without compromising the physiological functions of MYOCD signaling [43] as a part of the adaptive response of the heart to stress.

To the best of our knowledge, the role of MRTF-A and MRTF-B in failing myocardium was not evaluated in either study. Nevertheless, the data from experiments with MrtfA null mice strongly suggest that modulation of Mrtf-A expression in hypertrophy-induced [45] or post-MI [69] failing myocardium could be promising from therapeutic point of view. Of note, mRNA splicing of the MRTF- $B$ gene was altered in myocardial samples from patients with ischemic cardiomyopathy undergoing heart transplantation [93].

\section{Conclusion and Expectations}

Currently, a majority of the gene therapy assays are conducted using a single-candidate gene approach. There are two main strategies applied to the search for target genes for the treatment of HF. One is identification of terminal effector genes involved in the advanced and end-stage HF in patients. Another is to establish a new target for HF, searching for members of gene regulatory circuits, each of which can play a role in the control of a branch of terminal effector genes in the network, in both normal and diseased heart. Given the plethora of genes regulated by partnership of SRF and members of the MYOCD family in muscle cells, this balanced regulatory network plays a central role in normal heart development and adult heart function, whereas dysregulation of SRF/MYOCD/MRTF-dependent gene expression contributes to numerous disease models of the cardiovascular system.

Experimental manipulation of expression of MYOCD family genes has allowed the development of new animal models to study the mechanisms of DCM (MYOCD), cardiac hypertrophy, post-MI fibrosis (MRTF-A), and congenital heart disease (MRTF-B). Knowledge gained from these studies will guide the development of novel therapies for the treatment and prevention of HF development. Mice with a cardiomyocyte-specific deficiency in Myocd could aid in the development of apoptosis-blocking therapies for HF. Mrtf-A null models indicate that Mrtf-A inhibition might be therapeutically beneficial in post-MI cardiomyopathy settings. A normalization of activated MYOCD signaling in ventricular myocardium at midstages of HF development can improve impaired ventricular function.

Several cardiovascular-enriched microRNAs (miRNAs), both downstream and upstream to Myocd, were found to be involved in the SRF/MYOCD regulatory network [94-98]. In this sense, we suggest a promising therapeutic role for miRNA mimics/inhibitors to modify exaggerated MYOCD signaling in HF settings. In fact, recent data revealed that miR-9 can suppress Myocd translational activity in vitro and administration of the miR-9 mimic can attenuate cardiac hypertrophy remodeling in vivo [97]. 
Finally, an understanding of synergistic or additive interactions between MYOCD family factors in principal cell types of myocardium (myocytes and fibroblasts) is crucial before any therapeutic intervention could be entertained in preclinical trials.

\section{Acknowledgment}

Funding in the authors' laboratory was provided by the Spanish Biomedicine Research Program (SAF).

\section{References}

[1] T. A. McDonagh, M. Komajda, A. P. Maggioni, F. Zannad, and M. Gheorghiade, "Clinical trials in acute heart failure: simpler solutions to complex problems. Consensus document arising from a European Society of Cardiology cardiovascular roundtable think tank on acute heart failure, 12 May 2009," European Journal of Heart Failure, vol. 13, no. 12, pp. 1253-1260, 2011.

[2] J. O. Mudd and D. A. Kass, "Tackling heart failure in the twenty-first century," Nature, vol. 451, no. 7181, pp. 919-928, 2008.

[3] L. E. Vinge, P. W. Raake, and W. J. Koch, "Gene therapy in heart failure," Circulation Research, vol. 102, no. 12, pp. 14581470, 2008.

[4] K. B. Margulies, D. P. Bednarik, and D. L. Dries, "Genomics, transcriptional profiling, and heart failure," Journal of the American College of Cardiology, vol. 53, no. 19, pp. 1752-1759, 2009.

[5] S. P. Barry and P. A. Townsend, "What causes a broken heartmolecular insights into heart failure," International Review of Cell and Molecular Biology, vol. 284, no. C, pp. 113-179, 2010.

[6] M. G. Katz, A. S. Fargnoli, C. E. Tomasulo, L. A. Pritchette, and C. R. Bridges, "Model-specific selection of molecular targets for heart failure gene therapy," Journal of Gene Medicine, vol. 13, no. 10, pp. 573-586, 2011.

[7] F. I. Malik, J. J. Hartman, K. A. Elias et al., "Cardiac myosin activation: a potential therapeutic approach for systolic heart failure," Science, vol. 331, no. 6023, pp. 1439-1443, 2011.

[8] K. R. Chien and E. N. Olson, "Converging pathways and principles in heart development and disease: CV@CSH," Cell, vol. 110, no. 2, pp. 153-162, 2002.

[9] E. N. Olson, "Gene regulatory networks in the evolution and development of the heart," Science, vol. 313, no. 5795, pp. 1922-1927, 2006.

[10] B. J. D. Boukens, V. M. Christoffels, R. Coronel, and A. F. M. Moorman, "Developmental basis for electrophysiological heterogeneity in the ventricular and outflow tract myocardium as a substrate for life-threatening ventricular arrhythmias," Circulation Research, vol. 104, no. 1, pp. 19-31, 2009.

[11] A. T. Mikhailov and M. Torrado, Shaping the Heart in Development and Disease, Transworld Research Network, Kerala, India, 2010.

[12] D. Lai, X. Liu, A. Forrai et al., "Neuregulin 1 sustains the gene regulatory network in both trabecular and nontrabecular myocardium," Circulation Research, vol. 107, no. 6, pp. 715727, 2010.

[13] S. Yuasa, T. Onizuka, K. Shimoji et al., "Zac1 is an essential transcription factor for cardiac morphogenesis," Circulation Research, vol. 106, no. 6, pp. 1083-1091, 2010.
[14] L. Qian, J. D. Wythe, J. Liu et al., "Tinman/Nkx2-5 acts via miR-1 and upstream of Cdc42 to regulate heart function across species," Journal of Cell Biology, vol. 193, no. 7, pp. 11811196, 2011.

[15] J. Schlesinger, M. Schueler, M. Grunert et al., "The cardiac transcription network modulated by gata 4 , mef2a, nkx2.5, srf, histone modifications, and microRNAs," PLoS Genetics, vol. 7, no. 2, Article ID e1001313, 2011.

[16] F. E. Dewey, M. V. Perez, M. T. Wheeler et al., "Gene coexpression network topology of cardiac development, hypertrophy, and failure," Circulation, vol. 4, no. 1, pp. 26-35, 2011.

[17] J. M. Miano, "Serum response factor: toggling between disparate programs of gene expression," Journal of Molecular and Cellular Cardiology, vol. 35, no. 6, pp. 577-593, 2003.

[18] J. M. Miano, "Role of serum response factor in the pathogenesis of disease," Laboratory Investigation, vol. 90, no. 9, pp. 1274-1284, 2010.

[19] X. Zhang, G. Azhar, S. Helms et al., "Identification of new SRF binding sites in genes modulated by SRF over-expression in mouse hearts," Gene Regulation and Systems Biology, vol. 5, pp. 41-59, 2011.

[20] D. Z. Wang and E. N. Olson, "Control of smooth muscle development by the myocardin family of transcriptional coactivators," Current Opinion in Genetics and Development, vol. 14, no. 5, pp. 558-566, 2004.

[21] N. Liu and E. N. Olson, "Coactivator control of cardiovascular growth and remodeling," Current Opinion in Cell Biology, vol. 18, no. 6, pp. 715-722, 2006.

[22] E. N. Olson and A. Nordheim, "Linking actin dynamics and gene transcription to drive cellular motile functions," Nature Reviews Molecular Cell Biology, vol. 11, no. 5, pp. 353-365, 2010.

[23] R. J. Schwartz, "Serum response factor and co-factors, roles in cardiac development," in Heart Development and Regeneration, N. Rosenthal and R. P. Harvey, Eds., pp. 617-649, Academic Press, New York, NY, USA, 2010.

[24] M. S. Parmacek, "Myocardin-related transcription factors: critical coactivators regulating cardiovascular development and adaptation," Circulation Research, vol. 100, no. 5, pp. 633644, 2007.

[25] J. E. Kontaraki, F. I. Parthenakis, A. P. Patrianakos, I. K. Karalis, and P. E. Vardas, "Altered expression of early cardiac marker genes in circulating cells of patients with hypertrophic cardiomyopathy," Cardiovascular Pathology, vol. 16, no. 6, pp. 329-335, 2007.

[26] J. E. Kontaraki, M. E. Marketou, E. A. Zacharis, F. I. Parthenakis, and P. E. Vardas, "Early cardiac gene transcript levels in peripheral blood mononuclear cells in patients with untreated essential hypertension," Journal of Hypertension, vol. 29, no. 4, pp. 791-797, 2011.

[27] B. Cen, A. Selvaraj, and R. Prywes, "Myocardin/MKL family of SRF coactivators: key regulators of immediate early and muscle specific gene expression," Journal of Cellular Biochemistry, vol. 93, no. 1, pp. 74-82, 2004.

[28] G. C. T. Pipes, E. E. Creemers, and E. N. Olson, "The myocardin family of transcriptional coactivators: versatile regulators of cell growth, migration, and myogenesis," Genes and Development, vol. 20, no. 12, pp. 1545-1556, 2006.

[29] G. Posern and R. Treisman, “Actin' together: serum response factor, its cofactors and the link to signal transduction," Trends in Cell Biology, vol. 16, no. 11, pp. 588-596, 2006. 
[30] D. Z. Wang, P. S. Chang, Z. Wang et al., "Activation of cardiac gene expression by myocardin, a transcriptional cofactor for serum response factor," Cell, vol. 105, no. 7, pp. 851-862, 2001.

[31] D. Z. Wang, S. Li, D. Hockemeyer et al., "Potentiation of serum response factor activity by a family of myocardin-related transcription factors," Proceedings of the National Academy of Sciences of the United States of America, vol. 99, no. 23, pp. 14855-14860, 2002.

[32] E. E. Creemers, L. B. Sutherland, J. Oh, A. C. Barbosa, and E. N. Olson, "Coactivation of MEF2 by the SAP Domain Proteins Myocardin and MASTR," Molecular Cell, vol. 23, no. 1, pp. 8396, 2006.

[33] C. Wang, D. Cao, Q. Wang, and D. Z. Wang, "Synergistic activation of cardiac genes by myocardin and Tbx5," PLoS One, vol. 6, no. 8, Article ID e24242, 2011.

[34] Z. P. Liu, Z. Wang, H. Yanagisawa, and E. N. Olson, "Phenotypic modulation of smooth muscle cells through interaction of Foxo4 and Myocardin," Developmental Cell, vol. 9, no. 2, pp. 261-270, 2005.

[35] J. Oh, Z. Wang, D. Z. Wang, C. L. Lien, W. Xing, and E. N. Olson, "Target gene-specific modulation of myocardin activity by GATA transcription factors," Molecular and Cellular Biology, vol. 24, no. 19, pp. 8519-8528, 2004.

[36] Q. Sun, S. Taurin, N. Sethakorn et al., "Myocardin-dependent activation of the CArG box-rich smooth muscle $\gamma$-actin gene: preferential utilization of a single CArG element through functional association with the NKX3.1 homeodomain protein," Journal of Biological Chemistry, vol. 284, no. 47, pp. 3258232590, 2009.

[37] K. Kuwahara, T. Barrientos, G. C. T. Pipes, S. Li, and E. N. Olson, "Muscle-specific signaling mechanism that links actin dynamics to serum response factor," Molecular and Cellular Biology, vol. 25, no. 8, pp. 3173-3181, 2005.

[38] S. Guettler, M. K. Vartiainen, F. Miralles, B. Larijani, and R. Treisman, "RPEL motifs link the serum response factor cofactor MAL but not myocardin to Rho signaling via actin binding," Molecular and Cellular Biology, vol. 28, no. 2, pp. 732-742, 2008.

[39] S. Nakamura, K. Hayashi, K. Iwasaki et al., "Nuclear import mechanism for myocardin family members and their correlation with vascular smooth muscle cell phenotype," Journal of Biological Chemistry, vol. 285, no. 48, pp. 37314-37323, 2010.

[40] R. Pawłowski, E. K. Rajakylä, M. K. Vartiainen, and R. Treisman, "An actin-regulated importin $\alpha / \beta$-dependent extended bipartite NLS directs nuclear import of MRTF-A," EMBO Journal, vol. 29, no. 20, pp. 3448-3458, 2010.

[41] M. H. Mokalled, A. N. Johnson, E. E. Creemers, and E. N. Olson, "MASTR directs MyoD-dependent satellite cell differentiation during skeletal muscle regeneration," Genes and Development, vol. 26, no. 2, pp. 190-202, 2012.

[42] S. Li, D. Z. Wang, Z. Wang, J. A. Richardson, and E. N. Olson, "The serum response factor coactivator myocardin is required for vascular smooth muscle development," Proceedings of the National Academy of Sciences of the United States of America, vol. 100, no. 16, pp. 9366-9370, 2003.

[43] J. Huang, M. M. Lu, L. Cheng et al., "Myocardin is required for cardiomyocyte survival and maintenance of heart function," Proceedings of the National Academy of Sciences of the United States of America, vol. 106, no. 44, pp. 18734-18739, 2009.

[44] S. Li, S. Chang, X. Qi, J. A. Richardson, and E. N. Olson, "Requirement of a myocardin-related transcription factor for development of mammary myoepithelial cells," Molecular and Cellular Biology, vol. 26, no. 15, pp. 5797-5808, 2006.
[45] K. Kuwahara, H. Kinoshita, Y. Kuwabara et al., "Myocardinrelated transcription factor $\mathrm{A}$ is a common mediator of mechanical stress- and neurohumoral stimulation-induced cardiac hypertrophic signaling leading to activation of brain natriuretic peptide gene expression," Molecular and Cellular Biology, vol. 30, no. 17, pp. 4134-4148, 2010.

[46] Y. Sun, K. Boyd, W. Xu et al., "Acute myeloid leukemiaassociated Mkl1 (Mrtf-a) is a key regulator of mammary gland function," Molecular and Cellular Biology, vol. 26, no. 15, pp. 5809-5826, 2006.

[47] J. Oh, J. A. Richardson, and E. N. Olson, "Requirement of myocardin-related transcription factor-B for remodeling of branchial arch arteries and smooth muscle differentiation," Proceedings of the National Academy of Sciences of the United States of America, vol. 102, no. 42, pp. 15122-15127, 2005.

[48] J. Li, X. Zhu, M. Chen et al., "Myocardin-related transcription factor B is required in cardiac neural crest for smooth muscle differentiation and cardiovascular development," Proceedings of the National Academy of Sciences of the United States of America, vol. 102, no. 25, pp. 8916-8921, 2005.

[49] K. Wei, N. Che, and F. Chen, "Myocardin-related transcription factor B is required for normal mouse vascular development and smooth muscle gene expression," Developmental Dynamics, vol. 236, no. 2, pp. 416-425, 2007.

[50] M. H. Hoofnagle, R. L. Neppl, E. L. Berzin et al., "Myocardin is differentially required for the development of smooth muscle cells and cardiomyocytes," American Journal of Physiology, vol. 300, no. 5, pp. H1707-H1721, 2011.

[51] K. Kuwahara and K. Nakao, "New molecular mechanisms for cardiovascular disease: transcriptional pathways and novel therapeutic targets in heart failure," Journal of Pharmacological Sciences, vol. 116, no. 4, pp. 337-342, 2011.

[52] M. Hoshijima and K. R. Chien, "Mixed signals in heart failure: cancer rules," Journal of Clinical Investigation, vol. 109, no. 7, pp. 849-855, 2002.

[53] H. Akazawa and I. Komuro, "Roles of cardiac transcription factors in cardiac hypertrophy," Circulation Research, vol. 92, no. 10, pp. 1079-1088, 2003.

[54] E. N. Olson, "A decade of discoveries in cardiac biology," Nature Medicine, vol. 10, no. 5, pp. 467-474, 2004.

[55] T. Oka, J. Xu, and J. D. Molkentin, "Re-employment of developmental transcription factors in adult heart disease," Seminars in Cell and Developmental Biology, vol. 18, no. 1, pp. 117-131, 2007.

[56] M. Torrado, E. López, A. Centeno, C. Medrano, A. CastroBeiras, and A. T. Mikhailov, "Myocardin mRNA is augmented in the failing myocardium: expression profiling in the porcine model and human dilated cardiomyopathy," Journal of Molecular Medicine, vol. 81, no. 9, pp. 566-577, 2003.

[57] X. Zhang, G. Azhar, Y. Zhong, and J. Y. Wei, "Identification of a novel serum response factor cofactor in cardiac gene regulation," Journal of Biological Chemistry, vol. 279, no. 53, pp. 55626-55632, 2004.

[58] W. Xing, T. C. Zhang, D. Cao et al., "Myocardin induces cardiomyocyte hypertrophy," Circulation Research, vol. 98, no. 8, pp. 1089-1097, 2006.

[59] C. Badorff, F. H. Seeger, A. M. Zeiher, and S. Dimmeler, "Glycogen synthase kinase $3 \beta$ inhibits myocardin-dependent transcription and hypertrophy induction through site-specific phosphorylation," Circulation Research, vol. 97, no. 7, pp. 645654, 2005.

[60] W. Q. Tan, K. Wang, D. Y. Lv, and P. F. Li, "Foxo3a inhibits cardiomyocyte hypertrophy through transactivating catalase," 
Journal of Biological Chemistry, vol. 283, no. 44, pp. 2973029739, 2008.

[61] C. Z. Chiu, B. W. Wang, T. H. Chung, and K. G. Shyu, "Angiotensin II and the ERK pathway mediate the induction of myocardin by hypoxia in cultured rat neonatal cardiomyocytes," Clinical Science, vol. 119, no. 7, pp. 273-282, 2010.

[62] K. Wang, B. Long, J. Zhou, and P. F. Li, "miR-9 and NFATc3 regulate myocardin in cardiac hypertrophy," Journal of Biological Chemistry, vol. 285, no. 16, pp. 11903-11912, 2010.

[63] X. H. Liao, N. Wang, Q. X. Liu et al., "Myocardin-related transcription factor-A induces cardiomyocyte hypertrophy," IUBMB Life, vol. 63, no. 1, pp. 54-61, 2011.

[64] C. Z. Chiu, B. W. Wang, and K. G. Shyu, "Use of atorvastatin to inhibit hypoxia-induced myocardin expression," European Journal of Clinical Investigation, vol. 42, no. 5, pp. 564-571, 2012.

[65] J. Van Tuyn, S. Knaän-Shanzer, M. J. M. Van De Watering et al., "Activation of cardiac and smooth muscle-specific genes in primary human cells after forced expression of human myocardin," Cardiovascular Research, vol. 67, no. 2, pp. 245255, 2005.

[66] M. Milyavsky, I. Shats, A. Cholostoy et al., "Inactivation of myocardin and p16 during malignant transformation contributes to a differentiation defect," Cancer Cell, vol. 11, no. 2, pp. 133-146, 2007.

[67] R. H. Tang, X. L. Zheng, T. E. Callis et al., "Myocardin inhibits cellular proliferation by inhibiting NF- $\kappa \mathrm{B}(\mathrm{p} 65)$-dependent cell cycle progression," Proceedings of the National Academy of Sciences of the United States of America, vol. 105, no. 9, pp. 3362-3367, 2008.

[68] D. Shaposhnikov, A. Descot, J. Schilling, and G. Posern, "Myocardin-related transcription factor A regulates expression of Bok and Noxa and is involved in apoptotic signalling," Cell Cycle, vol. 11, no. 1, pp. 141-150, 2011.

[69] E. M. Small, J. E. Thatcher, L. B. Sutherland et al., "Myocardinrelated transcription factor-a controls myofibroblast activation and fibrosis in response to myocardial infarction," Circulation Research, vol. 107, no. 2, pp. 294-304, 2010.

[70] S. Ohnishi, H. Sumiyoshi, S. Kitamura, and N. Nagaya, "Mesenchymal stem cells attenuate cardiac fibroblast proliferation and collagen synthesis through paracrine actions," FEBS Letters, vol. 581, no. 21, pp. 3961-3966, 2007.

[71] Y. Wang, A. Sun, J. Xue, and Y. Jiang, "Adenovirus-mediated expression of hypoxia-inducible factor 1alpha double mutant converts neonatal cardiac fibroblasts into (cardio)myocyte phenotype," Cell Biochemistry and Function, vol. 30, no. 1, pp. 24-32, 2012.

[72] J. Chen, C. M. Kitchen, J. W. Streb, and J. M. Miano, "Myocardin: a component of a molecular switch for smooth muscle differentiation," Journal of Molecular and Cellular Cardiology, vol. 34, no. 10, pp. 1345-1356, 2002.

[73] T. Yoshida, K. Kawai-Kowase, and G. K. Owens, "Forced expression of myocardin is not sufficient for induction of smooth muscle differentiation in multipotential embryonic cells," Arteriosclerosis, Thrombosis, and Vascular Biology, vol. 24, no. 9, pp. 1596-1601, 2004.

[74] J. Wang, A. Li, Z. Wang, X. Feng, E. N. Olson, and R. J. Schwartz, "Myocardin sumoylation transactivates cardiogenic genes in pluripotent 10T1/2 fibroblasts," Molecular and Cellular Biology, vol. 27, no. 2, pp. 622-632, 2007.

[75] Z. Wang, D. Z. Wang, G. C. T. Pipes, and E. N. Olson, "Myocardin is a master regulator of smooth muscle gene expression," Proceedings of the National Academy of Sciences of the United States of America, vol. 100, no. 12, pp. 7129-7134, 2003.

[76] X. Long, R. D. Bell, W. T. Gerthoffer, B. V. Zlokovic, and J. M. Miano, "Myocardin is sufficient for a smooth musclelike contractile phenotype," Arteriosclerosis, Thrombosis, and Vascular Biology, vol. 28, no. 8, pp. 1505-1510, 2008.

[77] J. Van Tuyn, D. E. Atsma, E. M. Winter et al., "Epicardial cells of human adults can undergo an epithelial-to-mesenchymal transition and obtain characteristics of smooth muscle cells in vitro," Stem Cells, vol. 25, no. 2, pp. 271-278, 2007.

[78] J. Van Tuyn, D. A. Pijnappels, A. A. F. De Vries et al., "Fibroblasts from human postmyocardial infarction scars acquire properties of cardiomyocytes after transduction with a recombinant myocardin gene," FASEB Journal, vol. 21, no. 12, pp. 3369-3379, 2007.

[79] R. W. Grauss, J. Van Tuyn, P. Steendijk et al., "Forced myocardin expression enhances the therapeutic effect of human mesenchymal stem cells after transplantation in ischemic mouse hearts," Stem Cells, vol. 26, no. 4, pp. 1083-1093, 2008.

[80] X. Long and J. M. Miano, "Transforming growth factor-beta1 (TGF-beta1) utilizes distinct pathways for the transcriptional activation of microRNA 143/145 in human coronary artery smooth muscle cells," Journal of Biological Chemistry, vol. 286, no. 34, pp. 30119-30129, 2011.

[81] P. Qiu, R. P. Ritchie, Z. Fu et al., "Myocardin enhances Smad3-mediated transforming growth factor- $\beta 1$ signaling in a CArG box-independent manner: smad-binding element is an important cis element for SM22 $\alpha$ transcription in vivo," Circulation Research, vol. 97, no. 10, pp. 983-991, 2005.

[82] W. B. Xie, Z. Li, J. M. Miano, X. Long, and S. Y. Chen, "Smad3mediated myocardin silencing: a novel mechanism governing the initiation of smooth muscle differentiation," Journal of Biological Chemistry, vol. 286, no. 17, pp. 15050-15057, 2011.

[83] M. Dobaczewski, W. Chen, and N. G. Frangogiannis, "Transforming growth factor (TGF)- $\beta$ signaling in cardiac remodeling," Journal of Molecular and Cellular Cardiology, vol. 51, pp. 600-606, 2011.

[84] B. J. Crider, G. M. Risinger, C. J. Haaksma, E. W. Howard, and J. J. Tomasek, "Myocardin-related transcription factors A and $\mathrm{B}$ are key regulators of TGF- $\beta 1$-induced fibroblast to myofibroblast differentiation," Journal of Investigative Dermatology, vol. 131, pp. 2378-2385, 2011.

[85] M. S. Parmacek, "Myocardin-related transcription factor-a: mending a broken heart," Circulation Research, vol. 107, no. 2, pp. 168-170, 2010.

[86] M. Torrado, A. Centeno, E. López, and A. T. Mikhailov, "In vivo forced expression of myocardin in ventricular myocardium transiently impairs systolic performance in early neonatal pig heart," International Journal of Developmental Biology, vol. 53, no. 8-10, pp. 1457-1467, 2009.

[87] M. Torrado, R. Iglesias, B. Nespereira, and A. T. Mikhailov, "Identification of candidate genes potentially relevant to chamber-specific remodeling in postnatal ventricular myocardium," Journal of Biomedicine and Biotechnology, vol. 2010, Article ID 603159, 10 pages, 2010.

[88] M. Torrado, E. López, A. Centeno, A. Castro-Beiras, and A. T. Mikhailov, "Left-right asymmetric ventricular expression of CARP in the piglet heart: regional response to experimental heart failure," European Journal of Heart Failure, vol. 6, no. 2, pp. 161-172, 2004.

[89] J. J. Leenders, W. J. Wijnen, M. Hiller et al., "Regulation of cardiac gene expression by KLF15, a repressor of myocardin 
activity," Journal of Biological Chemistry, vol. 285, no. 35, pp. 27449-27456, 2010.

[90] A. Parlakian, C. Charvet, B. Escoubet et al., "Temporally controlled onset of dilated cardiomyopathy through disruption of the srf gene in adult heart," Circulation, vol. 112, no. 19, pp. 2930-2939, 2005.

[91] M. Steenman, Y. W. Chen, M. Le Cunff et al., "Transcriptomal analysis of failing and nonfailing human hearts," Physiological Genomics, vol. 12, pp. 97-112, 2003.

[92] M. Torrado, R. Iglesias, A. Centeno, E. Lopez, and A. T. Mikhailov, "Targeted gene-silencing reveals the functional significance of myocardin signaling in the failing heart," PLoS One, vol. 6, no. 10, Article ID e26392, 2011.

[93] S. W. Kong, Y. W. Hu, J. W. K. Ho et al., "Heart failureassociated changes in RNA splicing of sarcomere genes," Circulation, vol. 3, no. 2, pp. 138-146, 2010.

[94] Y. Zhao, E. Samal, and D. Srivastava, "Serum response factor regulates a muscle-specific microRNA that targets Hand2 during cardiogenesis," Nature, vol. 436, no. 7048, pp. 214-220, 2005.

[95] M. Xin, E. M. Small, L. B. Sutherland et al., "MicroRNAs miR-143 and miR-145 modulate cytoskeletal dynamics and responsiveness of smooth muscle cells to injury," Genes and Development, vol. 23, no. 18, pp. 2166-2178, 2009.

[96] Y. Jiang, H. A. O. Yin, and X. L. Zheng, "MicroRNA-1 inhibits myocardin-induced contractility of human vascular smooth muscle cells," Journal of Cellular Physiology, vol. 225, no. 2, pp. 506-511, 2010.

[97] K. Wang, B. Long, J. Zhou, and P. F. Li, "miR-9 and NFATc3 regulate myocardin in cardiac hypertrophy," Journal of Biological Chemistry, vol. 285, no. 16, pp. 11903-11912, 2010.

[98] J. Chen, H. Yin, Y. Jiang et al., "Induction of MicroRNA-1 by myocardin in smooth muscle cells inhibits cell proliferation," Arteriosclerosis, Thrombosis, and Vascular Biology, vol. 31, no. 2, pp. 368-375, 2011. 

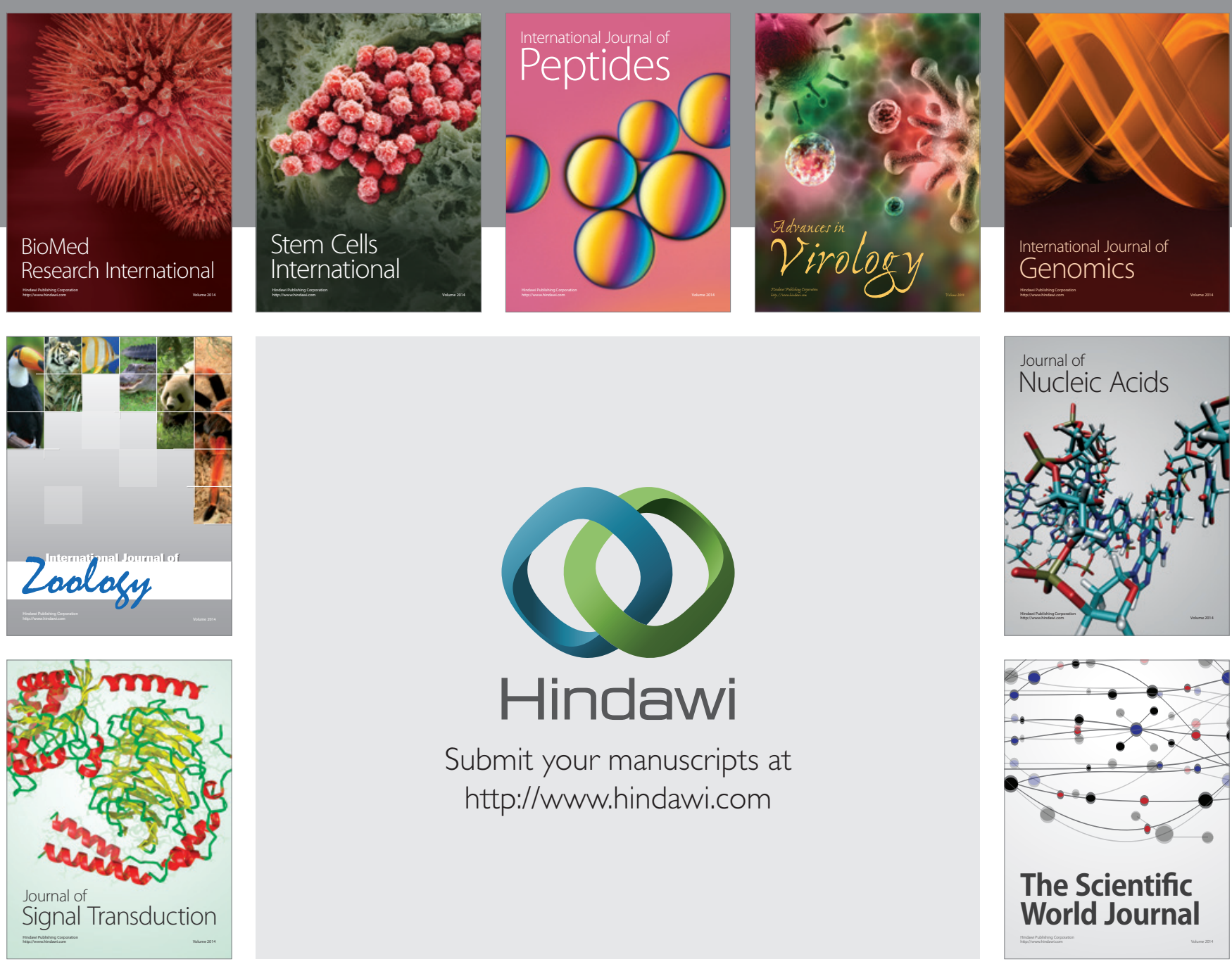

Submit your manuscripts at

http://www.hindawi.com
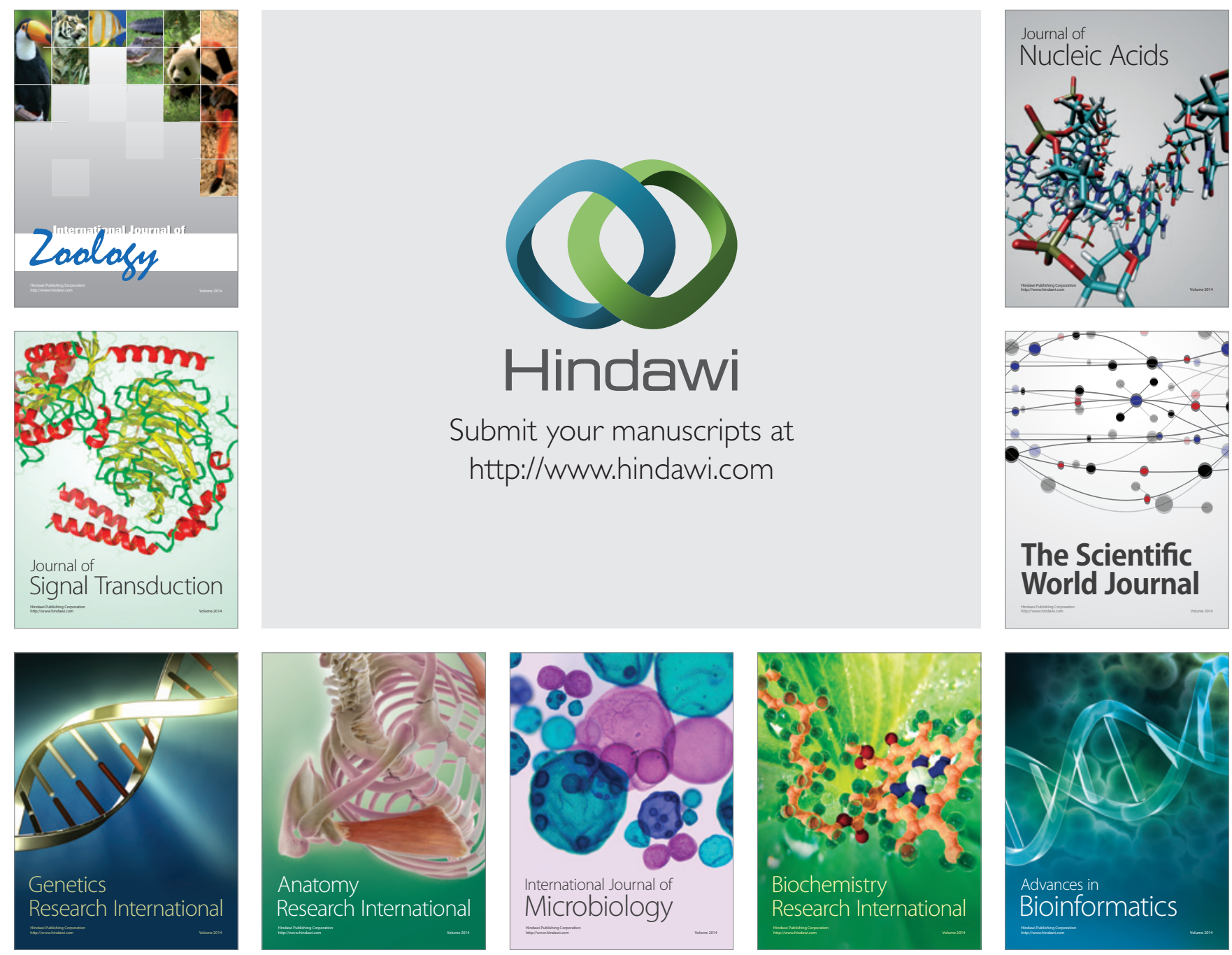

The Scientific World Journal
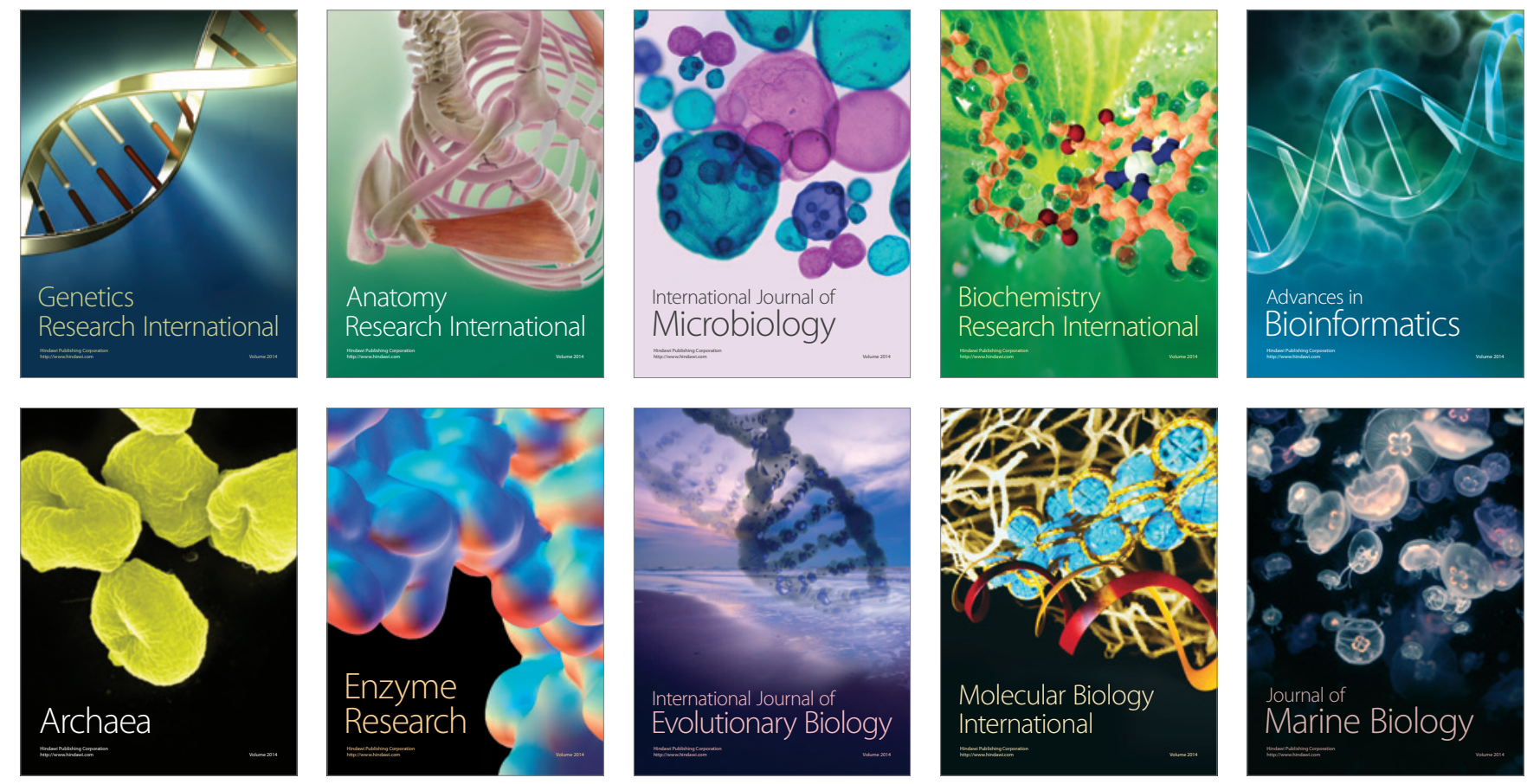